\title{
Handwritten Digit Recognition using Convolutional Neural Network in Python with Tensorflow and Observe the Variation of Accuracies for Various Hidden Layers
}

\author{
Fathma Siddique $^{1 \#}$, Shadman Sakib ${ }^{2 *}$, Md. Abu Bakr Siddique ${ }^{3 \$}$ \\ ${ }^{1}$ Department of CSE, International University of Business Agriculture and Technology, Dhaka 1230, Bangladesh \\ ${ }^{2,3}$ Department of EEE, International University of Business Agriculture and Technology, Dhaka 1230, Bangladesh \\ siddiquefathma@gmail.com\#, sakibshadman15@gmail.com and15305026@iubat.edu*, absiddique@iubat.edu\$ \\ Corresponding Author: 15305026@iubat.edu*
}

\begin{abstract}
In recent times, with the increase of Artificial Neural Network (ANN), deep learning has brought a dramatic twist in the field of machine learning by making it more Artificial Intelligence (AI). Deep learning is used remarkably used in vast ranges of fields because of its diverse range of applications such as surveillance, health, medicine, sports, robotics, drones etc. In deep learning, Convolutional Neural Network (CNN) is at the center of spectacular advances that mixes Artificial Neural Network (ANN) and up to date deep learning strategies. It has been used broadly in pattern recognition, sentence classification, speech recognition, face recognition, text categorization, document analysis, scene, and handwritten digit recognition. The goal of this paper is to observe the variation of accuracies of CNN to classify handwritten digits using various numbers of hidden layer and epochs and to make the comparison between the accuracies. For this performance evaluation of $\mathrm{CNN}$, we performed our experiment using Modified National Institute of Standards and Technology (MNIST) dataset. Further, the network is trained using stochastic gradient descent and the backpropagation algorithm.
\end{abstract}

Keywords-Handwritten digit recognition, Convolutional Neural Network (CNN), Deep learning, MNIST dataset, Epochs, Hidden Layers, Stochastic Gradient Descent, Backpropagation

\section{INTRODUCTION}

With time the numbers of fields are increasing in which deep learning can be applied. In deep learning, Convolutional Neural Networking (CNN) [1, 2] is being used for visual imagery analyzing. Object detection, face recognition, robotics, video analysis, segmentation, pattern recognition, natural language processing, spam detection, topic categorization, regression analysis, speech recognition, image classification are some of the examples that can be done using Convolutional Neural Networking. The accuracies in these fields including handwritten digits recognition using Deep Convolutional Neural Networks (CNNs) have reached human level perfection. Mammalian visual systems' biological model is the one by which the architecture of the CNN is inspired. Cells in the cat's visual cortex are sensitized to a tiny area of the visual field identified which is recognized as the receptive field [3]. It was found by D. H. Hubel et al. in 1062. The neocognitron [4], the pattern recognition model inspired by the work of D. H. Hubel et al. [5, 6] was the first computer vision. It was introduced by Fukushima in 1980. In 1998, the framework of CNNs is designed by LeCun et al. [7] which had seven layers of convolutional neural networks. It was adept in handwritten digits classification direct from pixel values of images [8]. Gradient descent and back propagation algorithm [9] is used for training the model. In handwritten recognition digits, characters are given as input. The model can be recognized by the system. A simple artificial neural network (ANN) has an input layer, an output layer and some hidden layers between the input and output layer. The CNN has a very similar architecture as ANN. There are several neurons in each layer in ANN. The weighted sum of all the neurons of a layer becomes the input of a neuron of next layer adding a biased value. In $\mathrm{CNN}$ the layer has three dimensions. Here all the neurons are not fully connected. Instead, every neuron in the layer is connected to the local receptive field. A cost function generates in order to train the network. It compares the output of the network with the desired output. The signal propagates back to the system, again and again, to update the shared weights and biases in all the receptive fields to minimize the value of cost function which increases the network's performance [10-12]. The goal of this article is to observe the influence of hidden layers of a CNN for handwritten digits. We have applied a different type of Convolutional Neural Network algorithm on Modified National Institute of Standards and Technology (MNIST) dataset using tensorflow, a Neural Network library written in python. The main purpose of this paper is to analyze the variation of outcome results for using a different combination of hidden layers of Convolutional Neural network. Stochastic gradient and backpropagation algorithm is used for training the network and the forward algorithm is used for testing.

\section{LITERATURE REVIEW}

$\mathrm{CNN}$ is playing an important role in many sectors like image processing. It has a powerful impact on many fields. Even, in nano-technologies like manufacturing semiconductors, $\mathrm{CNN}$ is used for fault detection and classification [13]. Handwritten digit recognition has become an issue of interest among researchers. There are a large number of papers and articles are being published these days about this topic. In research it is shown that Deep Learning algorithm like multilayer CNN using Keras with Theano and Tensorflow gives the highest accuracy in comparison with the most widely used machine learning 
algorithms like SVM, KNN \& RFC. Because of its highest accuracy, Convolutional Neural Network (CNN) is being used in a large scale in image classification, video analysis etc. Many researchers are trying to make sentiment recognition in a sentence. $\mathrm{CNN}$ is being used in natural language processing and sentiment recognition by varying different parameters [14]. It is pretty challenging to get a good performance as more parameters are needed for the large-scale neural network. Many researchers are trying to increase the accuracy with less error in CNN. In another research, they have shown that deep nets perform better when they are trained by simple back-propagation. Their architecture results in the lowest error rate on MNIST compare to NORB and CIFAR10 [15]. Researchers are working on this issue to reduce the error rate as much as possible in handwriting recognition. In one research, an error rate of $1.19 \%$ is achieved using 3-NN trained and tested on MNIST [22]. Deep CNN can be adjustable with the input image noise [16]. Coherence recurrent convolutional network (CRCN) is a multimodal neural architecture [17]. It is being used in recovering sentences in an image. Some researchers are trying to come up with new techniques to avoid drawbacks of traditional convolutional layer's. Ncfm (No combination of feature maps) is a technique which can be applied for better performance using MNIST datasets [18]. Its accuracy is $99.81 \%$ and it can be applied for largescale data. New applications of CNN are developing day by day with many kinds of research. Researchers are trying hard to minimize the error rates. Using MNIST datasets and CIFAR, error rates are being observed [19]. To clean blur images CNN is being used. For this purpose, a new model was proposed using MNIST dataset. This approach reaches an accuracy of $98 \%$ and loss range $0.1 \%$ to $8.5 \%$ [20]. In Germany, a traffic sign recognition model of CNN is suggested. It proposed a faster performance with $99.65 \%$ accuracy [21]. Loss function was designed, which is applicable for light-weighted $1 \mathrm{D}$ and 2D CNN. In this case the accuracies were $93 \%$ and $91 \%$ respectively $[22,23]$.

\section{Modeling OF Convolutional Neural NeTwork to Classify HaNDWRITTEN DigITS}

To recognize the handwritten digits, a seven-layered convolutional neural network with one input layer followed by five hidden layers and one output layer is designed and illustrated in figure 1 .

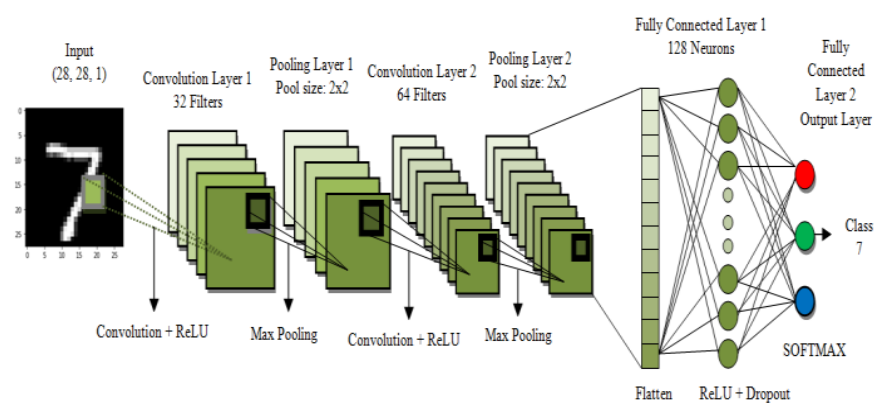

Fig. 1. A seven-layered convolutional neural network for digit recognition

The input layer consists of 28 by 28 pixel images which mean that the network contains 784 neurons as input data.
The input pixels are grayscale with a value 0 for a white pixel and 1 for a black pixel. Here, this model of CNN has five hidden layers. The first hidden layer is the convolution layer 1 which is responsible for feature extraction from an input data. This layer performs convolution operation to small localized areas by convolving a filter with the previous layer. In addition, it consists of multiple feature maps with learnable kernels and rectified linear units (ReLU). The kernel size determines the locality of the filters. ReLU is used as an activation function at the end of each convolution layer as well as a fully connected layer to enhance the performance of the model. The next hidden layer is the pooling layer 1 . It reduces the output information from the convolution layer and reduces the number of parameters and computational complexity of the model. The different types of pooling are max pooling, min pooling, average pooling, and L2 pooling. Here, max pooling is used to subsample the dimension of each feature map. Convolution layer 2 and pooling layer 2 which has the same function as convolution layer 1 and pooling layer 1 and operates in the same way except for their feature maps and kernel size varies. A Flatten layer is used after the pooling layer which converts the 2D featured map matrix to a $1 \mathrm{D}$ feature vector and allows the output to get handled by the fully connected layers. A fully connected layer is another hidden layer also known as the dense layer. It is similar to the hidden layer of Artificial Neural Networks (ANNs) but here it is fully connected and connects every neuron from the previous layer to the next layer. In order to reduce overfitting, dropout regularization method is used at fully connected layer 1 . It randomly switches off some neurons during training to improve the performance of the network by making it more robust. This causes the network to become capable of better generalization and less compelling to overfit the training data. The output layer of the network consists of ten neurons and determines the digits numbered from 0 to 9 . Since the output layer uses an activation function such as softmax, which is used to enhance the performance of the model, classifies the output digit from 0 through 9 which has the highest activation value.

The MNIST handwritten digits [24] database is used for the experiment. Out of 70,000 scanned images of handwritten digits from the MNIST database, 60,000 scanned images of digits are used for training the network and 10,000 scanned images of digits are used to test the network. The images that are used for training and testing the network all are the grayscale image with a size of $28 \times 28$ pixels. Character $x$ is used to represent a training input where $\mathrm{x}$ is a 784-dimensional vector as the input of $\mathrm{x}$ is regarded as $28 \times 28$ pixels. The equivalent desired output is expressed by $\mathrm{y}(\mathrm{x})$, where $\mathrm{y}$ is a 10-dimensional vector. The aim of the network is to find the convenient wights and biases so that the output of the network approximates $y(x)$ for all training inputs $x$ as it completely depends on weight values and bias values. To compute the network performances, a cost function is defined, expressed by equation 1 [25].

$$
C(w, b)=\frac{1}{2 n} \sum_{x}\left[y(x)-a^{2}\right]^{2}
$$

Where $\mathrm{w}$ is the cumulation of weights in the network, $\mathrm{b}$ is all the biases, $\mathrm{n}$ is the total number of training inputs and $\mathrm{a}$ is 
the actual output. The actual output a depends on $\mathrm{x}, \mathrm{w}$, and b. $\mathrm{C}(\mathrm{w}, \mathrm{b})$ is non-negative as all the terms in the sum is nonnegative. Moreover, $\mathrm{C}(\mathrm{w}, \mathrm{b})=0$, precisely when desired output $\mathrm{y}(\mathrm{x})$ is comparatively equal to the actual output, a, for all training inputs, $n$. To reduce the cost $\mathrm{C}(\mathrm{w}, \mathrm{b})$ to a smaller degree as a function of weight and biases, the training algorithm has to find a set of weight and biases which cause the cost to become as small as possible. This is done using an algorithm known as gradient descent. In other words, gradient descent is an optimization algorithm that twists its parameters iteratively to minimize a cost function to its local minimum. The gradient descent algorithm deploys the following equations [25] to set the weight and biases.

$$
\begin{aligned}
& w^{\text {new }}=w^{\text {old }}-\eta \frac{\partial C}{\partial w^{\text {old }}} \\
& b^{\text {new }}=b^{\text {old }}-\eta \frac{\partial C}{\partial b^{\text {old }}}
\end{aligned}
$$

And to attain the global minimum of the cost $\mathrm{C}(\mathrm{w}, \mathrm{b})$ shown in figure 2.

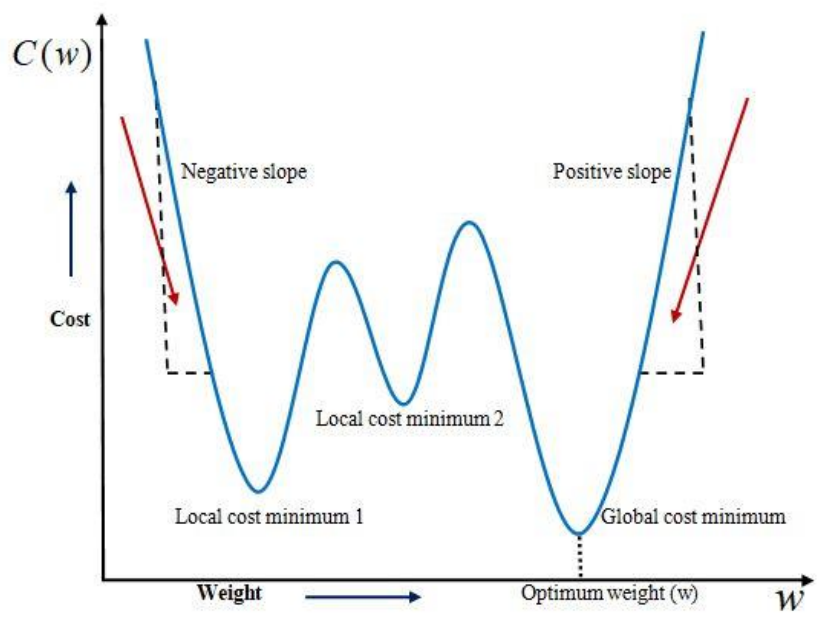

Fig. 2. Graphical Representation of Cost vs. Weight

However, the gradient descent algorithm may be unusable when the training data size is very large. Therefore, to enhance the performance of the network, a stochastic version of the algorithm is used. In Stochastic Gradient Descent (SDG) a small number of iteration will find effective solutions for the optimization problems. Moreover, in SDG, a small number of iteration will lead to a suitable solution. The Stochastic Gradient Descent algorithm utilizes the following equations [25]

$$
\begin{aligned}
w^{\text {new }} & =w^{\text {old }}-\frac{\eta}{m} \frac{\partial C_{x j}}{\partial w^{\text {old }}} \\
b^{\text {new }} & =b^{\text {old }}-\frac{\eta}{m} \frac{\partial C_{x j}}{\partial w^{\text {old }}}
\end{aligned}
$$

To find the amount of weight that contributes to the total error of the network Backpropagation method is used. The backpropagation of the network is illustrated by the following equations [25]

$$
\begin{gathered}
\delta^{L}=\frac{\partial C}{\partial a^{(L)}} \frac{\partial a^{(L)}}{\partial z^{(L)}}=\frac{1}{n}\left(a^{(L)}-1\right) f^{\prime}\left(z^{(L)}\right) \\
\delta^{l}=\frac{\partial C}{\partial z^{(l)}}=\frac{\partial C}{\partial z^{(l)}} \frac{\partial z^{(l+1)}}{\partial z^{(l)}}=\frac{\partial z^{(l+1)}}{\partial z^{(l)}} \delta^{l+1}=w^{l+1} \delta^{l+1} f^{\prime}\left(z^{l}\right)
\end{gathered}
$$

$$
\begin{aligned}
& \frac{\partial C}{\partial b^{(l)}}=\delta^{l} \\
& \frac{\partial C}{\partial w^{(L)}}=a^{l-1} \delta^{l}
\end{aligned}
$$

\section{MNIST DATASET}

Modified National Institute of Standards and Technology (MNIST) is a large set of computer vision dataset which is extensively used for training and testing different systems. It was created from the two special datasets of National Institute of Standards and Technology (NIST) which holds binary images of handwritten digits. The training set contains handwritten digits from 250 people, among them $50 \%$ training dataset was employees from the Census Bureau and the rest of it was from high school students [26]. However, it is often attributed as the first datasets among other datasets to prove the effectiveness of the neural networks.
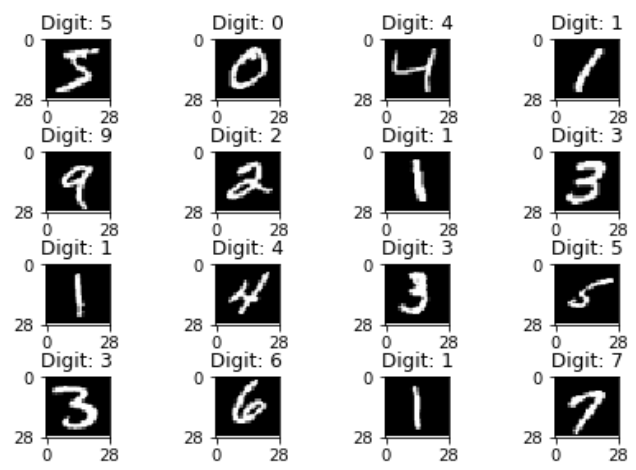

Fig. 3. Sample images of MNIST handwritten digit dataset

The database contains 60,000 images used for training as well as few of them can be used for cross-validation purposes and 10,000 images used for testing [27]. All the digits are grayscale and positioned in a fixed size where the intensity lies at the center of the image with $28 \times 28$ pixels. Since all the images are $28 \times 28$ pixels, it forms an array which can be flattened into $28 * 28=784$ dimensional vector. Each component of the vector is a binary value which describes the intensity of the pixel.

\section{Simulation RESUltS AND DiscUSSION}

In this section, CNN has been applied on the MNIST dataset in order to observe the variation of accuracies for handwritten digits. The accuracies are obtained using tensorflow in python. Training and validation accuracy for 15 different epochs were observed exchanging the hidden layers for various combinations of convolution and hidden layers by taking the batch size 100 for all the cases. Figure 4, 5, 6, 7, 8, and 9 shows the performance of CNN for different combinations of convolution and hidden layers.

In the first case shown in figure 4 , the first hidden layer is the convolutional layer 1 which is used for the feature extraction. It consists of 32 filters with the kernel size of $3 \times 3$ pixels and the rectified linear units (ReLU) is used as an activation function to enhance the performance. The next hidden layer is the convolutional layer 2 consists of 64 filters with a kernel size of $3 \times 3$ pixels and ReLU. Next, a pooling layer 1 is defined where max pooling is used with a pool size of $2 \times 2$ pixels to minimize the spatial size of the output of a 
convolution layer. A regularization layer dropout is used next to the pooling layer 1 where it randomly eliminates $25 \%$ of the neurons in the layer to reduce overfitting. A flatten layer is used after the dropout which converts the 2D filter matrix into $1 \mathrm{D}$ feature vector before entering into the fully connected layers. The next hidden layer used after the flatten layer is the fully connected layer 1 consists of 128 neurons and ReLU. A dropout with a probability of $50 \%$ is used after the fully connected layer 1. Finally, the output layer which is used here as fully connected layer 2 contains 10 neurons for 10 classes and determines the digits numbered from 0 to 9 .

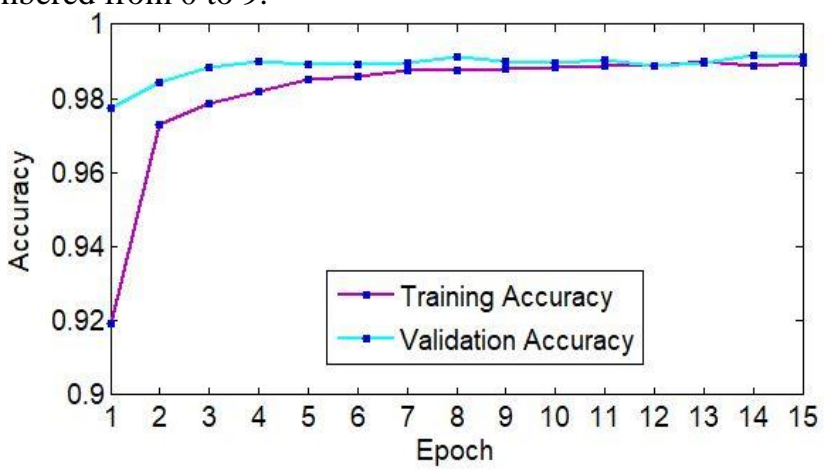

Fig. 4. Observed accuracy for case 1
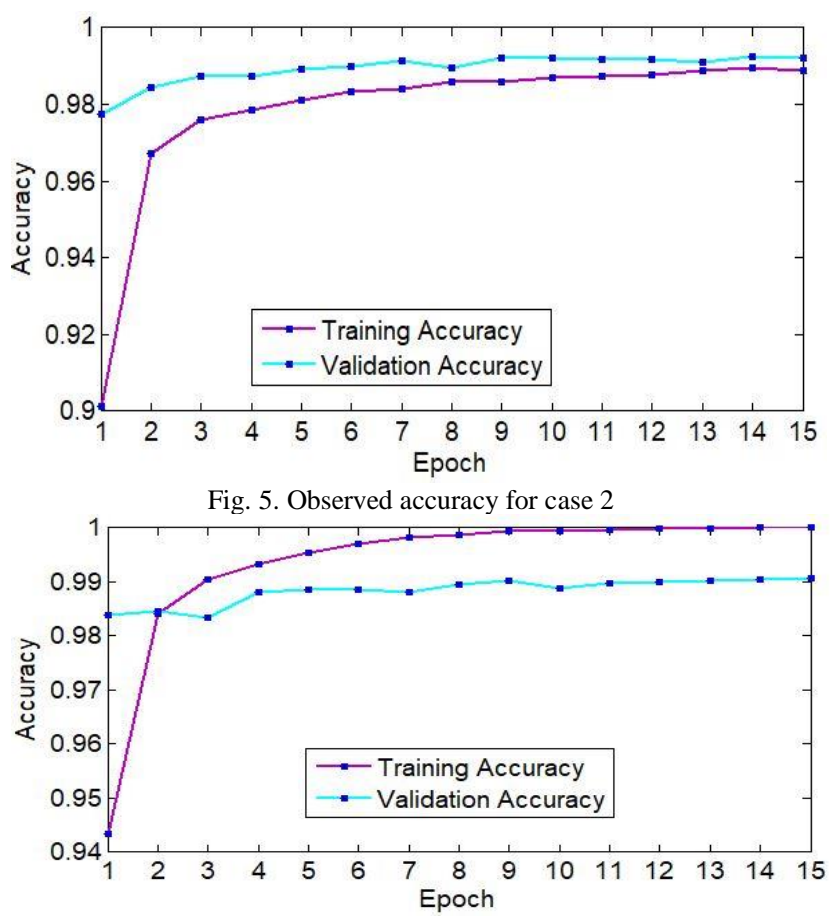

Fig. 6. Observed accuracy for case 3

A softmax activation function is incorporated with the output layer to output digit from 0 through 9 . The CNN is fit over 15 epochs with a batch size of 100 . The overall validation accuracy in the performance is found $99.11 \%$. At epoch 1 the minimum training accuracy of $91.94 \%$ is found and $97.73 \%$ of validation accuracy is found. At epoch 13, the maximum training accuracy is found $98.99 \%$ and at epoch 14, the maximum validation accuracy is found $99.16 \%$. The total test loss for this case is found approximately 0.037045 .

Figure 5 is defined for case 2 , where convolution 1 , pooling 1 and convolution 2 , pooling 2 is used one after another. A dropout is used followed by the flatten layer and fully connected layer 1 . Before the fully connected layer 2 another dropout is used. The dimensions and parameters used here and for the next cases are same which are used earlier for case 1 . The overall validation accuracy in the performance is found $99.21 \%$. At epoch 1 the minimum training and validation accuracy are found. The minimum training accuracy is $90.11 \%$ and the minimum validation accuracy is $97.74 \%$. The maximum training and validation accuracy are found at epoch 14 . The maximum training and validation accuracies are $98.94 \%$ and $99.24 \%$. The total test loss is found approximately 0.026303 .

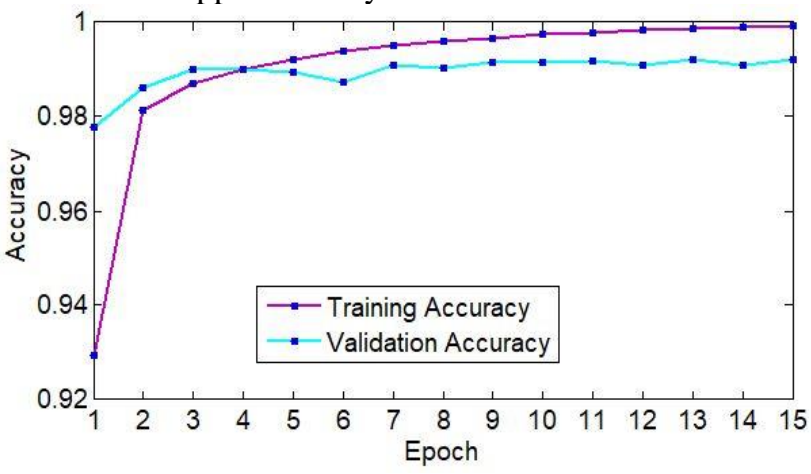

Fig. 7. Observed accuracy for case 4

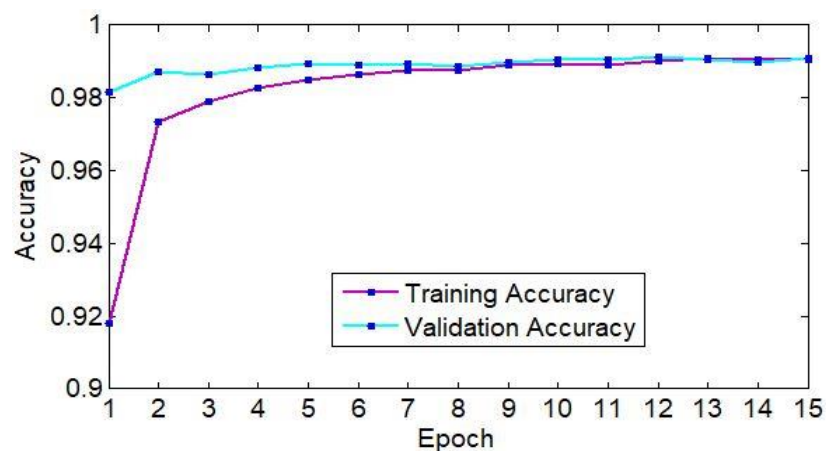

Fig. 8. Observed accuracy for case 5

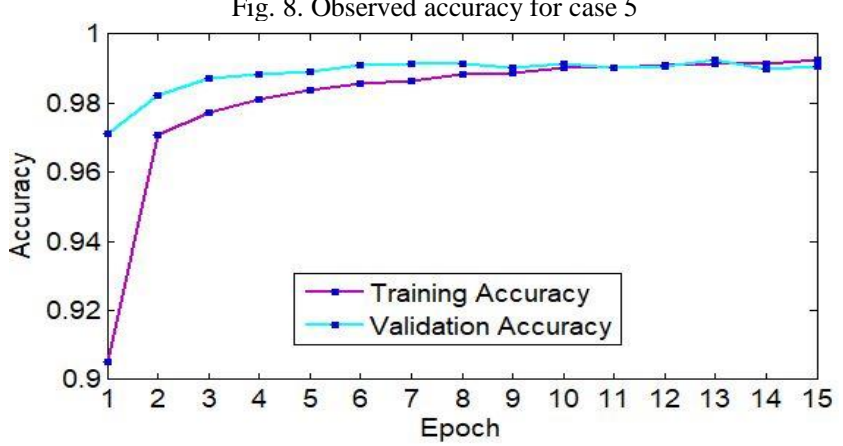

Fig. 9. Observed accuracy for case 6

For the case 3, shown in figure 6 , where two convolutions are taken one after another followed by a pooling layer. After the pooling layer, a flatten layer is used followed by the two fully connected layers without any dropout. The overall validation accuracy in the performance is found $99.06 \%$. The minimum training accuracy is found $94.35 \%$ at epoch 1 and at epoch 3 , the minimum validation accuracy is found $98.33 \%$. The maximum training and validation accuracies are 1\% and $99.06 \%$ found at epoch 15 . The total test loss is found approximately 0.049449 .

Similarly, in case 4 shown in figure 7 , convolution 1 , pooling 1 and convolution 2, pooling 2 are used alternately followed by a flatten layer and two fully connected layers 
without any dropout. The overall validation accuracy in the performance is found $99.20 \%$. At epoch 1 the minimum training and validation accuracy are found. The minimum training accuracy is $92.94 \%$ and the minimum validation accuracy is $97.79 \%$. The maximum training accuracy is found $99.92 \%$ at epoch 15 and at epoch 13, the maximum validation accuracy also found $99.92 \%$. The total test loss is found approximately 0.032287 .

Again, for case 5 shown in figure 8, two convolutions are used one after another followed by a pooling layer, flatten layer and fully connected layer 1. A dropout is used before the fully connected layer 2 . The overall validation accuracy in the performance is found $99.09 \%$. The minimum training and validation accuracy are found at epoch 1 . The minimum training accuracy is $91.80 \%$ and the minimum validation accuracy is $98.16 \%$. At epoch 13 , the maximum training accuracy is found $99.09 \%$ and the maximum validation accuracy is found $99.12 \%$ at epoch 12 . The total test loss is found approximately 0.034337 .

Finally, for the case 6 shown in figure 9, convolution 1, pooling 1 and convolution 2 , pooling 2 are used alternately followed by a flatten layer and fully connected layer 1 . A dropout is used before the fully connected layer 2 . The overall validation accuracy in the performance is found $99.07 \%$. At epoch 1 the minimum training and validation accuracy are found. The minimum training accuracy is $90.5 \%$ and the minimum validation accuracy is $97.13 \%$. The maximum training accuracy is found $99.24 \%$ at epoch 15 and the maximum validation accuracy is found $99.26 \%$ at epoch 13. The total test loss is found approximately 0.028596 .

\section{CONCLUSION}

In this paper, the variations of accuracies for handwritten digit were observed for 15 epochs by varying the hidden layers. The accuracy curves were generated for the six cases for the different parameter using CNN MNIST digit dataset. The maximum and minimum accuracies were observed for different hidden layers variation with a batch size of 100 . Among all the observation, the maximum accuracy in the performance was found $99.21 \%$ for 15 epochs in case 2 (Conv1, pool1, Conv2, pool2 with 2 dropouts). In digit recognition, this type of higher accuracy will cooperate to speed up the performance of the machine more adequately. However, the minimum accuracy among all observation in the performance was found $97.07 \%$ in case 6 (Conv1, pool1, Conv2, pool2 with 1 dropout). Moreover, among all the cases, the total highest test loss is approximately 0.049449 found in case 3 without dropout and the total lowest test loss is approximately 0.026303 found in case 2 with dropout. This low loss will provide CNN better performance to attain better image resolution and noise processing. In the future, our plan is to observe the variation in the overall classification accuracy by varying the number of hidden layers and batch size.

\section{REFERENCES}

Y. LeCun, B. Boser, J. S. Denker, D. Henderson, R. E. Howard, W. Hubbard, et al., "Backpropagation applied to handwritten zip code recognition," Neural computation, vol. 1, pp. 541-551, 1989. [2] A. Krizhevsky, I. Sutskever, and G. E. Hinton, "Imagenet
classification with deep convolutional neural networks," in Advances in neural information processing systems, 2012, pp. 1097-1105.
[3] D. Hubel and T. Wiesel, "Aberrant visual projections in the Siamese cat," The Journal of physiology, vol. 218, pp. 33-62, 1971.

[4] Y. LeCun, Y. Bengio, and G. Hinton, "Deep learning," nature, vol. 521, p. 436, 2015.

[5] D. Cireşan, U. Meier, and J. Schmidhuber, "Multi-column deep neural networks for image classification," arXiv preprint arXiv:1202.2745, 2012.

[6] K. Fukushima and S. Miyake, "Neocognitron: A self-organizing neural network model for a mechanism of visual pattern recognition," in Competition and cooperation in neural nets, ed: Springer, 1982, pp. 267-285.

[7] Y. LeCun, B. E. Boser, J. S. Denker, D. Henderson, R. E. Howard, W. E. Hubbard, et al., "Handwritten digit recognition with a backpropagation network," in Advances in neural information processing systems, 1990, pp. 396-404.

[8] Y. LeCun, L. Bottou, Y. Bengio, and P. Haffner, "Gradient-based learning applied to document recognition," Proceedings of the IEEE, vol. 86, pp. 2278-2324, 1998.

[9] R. Hecht-Nielsen, "Theory of the backpropagation neural network," in Neural networks for perception, ed: Elsevier, 1992, pp. 65-93.

[10] Y. LeCun, "LeNet-5, convolutional neural networks," URL: http://yann. lecun. com/exdb/lenet, vol. 20, 2015.

[11] S. J. Russell and P. Norvig, Artificial intelligence: a modern approach: Malaysia; Pearson Education Limited, 2016.

[12] S. Haykin, "Neural networks: A comprehensive foundation: MacMillan College," New York, 1994.

[13] K. B. Lee, S. Cheon, and C. O. Kim, "A convolutional neural network for fault classification and diagnosis in semiconductor manufacturing processes," IEEE Transactions on Semiconductor Manufacturing, vol. 30, pp. 135-142, 2017.

[14] K. G. Pasi and S. R. Naik, "Effect of parameter variations on accuracy of Convolutional Neural Network," in 2016 International Conference on Computing, Analytics and Security Trends (CAST), 2016, pp. 398-403.

[15] D. C. Ciresan, U. Meier, J. Masci, L. M. Gambardella, and J. Schmidhuber, "Flexible, high performance convolutional neural networks for image classification," in Twenty-Second International Joint Conference on Artificial Intelligence, 2011.

[16] K. Isogawa, T. Ida, T. Shiodera, and T. Takeguchi, "Deep shrinkage convolutional neural network for adaptive noise reduction," IEEE Signal Processing Letters, vol. 25, pp. 224-228, 2018.

[17] C. C. Park, Y. Kim, and G. Kim, "Retrieval of sentence sequences for an image stream via coherence recurrent convolutional networks," IEEE transactions on pattern analysis and machine intelligence, vol. 40, pp. 945-957, 2018.

[18] Y. Yin, J. Wu, and H. Zheng, "Ncfm: Accurate handwritten digits recognition using convolutional neural networks," in 2016 International Joint Conference on Neural Networks (IJCNN), 2016, pp. 525-531.

[19] L. Xie, J. Wang, Z. Wei, M. Wang, and Q. Tian, "Disturblabel: Regularizing cnn on the loss layer," in Proceedings of the IEEE Conference on Computer Vision and Pattern Recognition, 2016, pp. $4753-4762$.

[20] A. Tavanaei and A. S. Maida, "Multi-layer unsupervised learning in a spiking convolutional neural network," in 2017 International Joint Conference on Neural Networks (IJCNN), 2017, pp. 20232030.

[21] J. Jin, K. Fu, and C. Zhang, "Traffic sign recognition with hinge loss trained convolutional neural networks," IEEE Transactions on Intelligent Transportation Systems, vol. 15, pp. 1991-2000, 2014.

[22] M. Wu and Z. Zhang, "Handwritten digit classification using the mnist data set," Course project CSE802: Pattern Classification \& Analysis, 2010

[23] Y. Liu and Q. Liu, "Convolutional neural networks with largemargin softmax loss function for cognitive load recognition," in 2017 36th Chinese Control Conference (CCC), 2017, pp. 40454049.

[24] Y. LeCun, "The MNIST database of handwritten digits," http://yann. lecun. com/exdb/mnist/, 1998.

[25] M. A. Nielsen, Neural networks and deep learning vol. 25: Determination press USA, 2015.

[26] B. Zhang and S. N. Srihari, "Fast k-nearest neighbor classification using cluster-based trees," IEEE Transactions on Pattern analysis and machine intelligence, vol. 26, pp. 525-528, 2004. 
[27] E. Kussul and T. Baidyk, "Improved method of handwritten digit recognition tested on MNIST database," Image and Vision Computing, vol. 22, pp. 971-981, 2004. 